\title{
Deformed Harmonic Oscillators for Metal Clusters and Balian-Bloch Theory
}

\author{
Dennis Bonatsos*, D. Lenis*, P. P. Raychev ${ }^{\dagger}$, P. A. Terziev ${ }^{\dagger}$ \\ * Institute of Nuclear Physics, N.C.S.R. "Demokritos" \\ GR-15310 Aghia Paraskevi, Attiki, Greece \\ † Institute for Nuclear Research and Nuclear Energy, Bulgarian Academy of Sciences \\ 72 Tzarigrad Road, BG-1784 Sofia, Bulgaria
}

\begin{abstract}
The predictions for the shell structure of metal clusters of the three-dimensional $q$ deformed harmonic oscillator (3D q-HO), utilizing techniques of quantum groups and having the symmetry $\mathrm{u}_{q}(3) \supset \mathrm{so}_{q}(3)$, are compared to the restrictions imposed by the periodic orbit theory of Balian and Bloch, of electrons moving in a spherical cavity. It is shown that agreement between the predictions of the two models is established through the introduction of an additional term to the Hamiltonian of the 3D $q-\mathrm{HO}$, which does not influence the predictions for supershells. This term preserves the $\mathrm{u}_{q}(3) \supset \mathrm{so}_{q}(3)$ symmetry, while in addition it can be derived through a variational procedure, analogous to the one leading from the usual harmonic oscillator to the Morse oscillator by introducing the concept of the Variable Frequency Oscillator (VFO).
\end{abstract}

\section{Introduction}

Algebraic models are popular in several branches of physics [1, 2, In ideal cases, algebraic models are able to describe in good approximation several properties of a physical system using a limited number of appropriate (usually collective) degrees of freedom. In addition to providing successful predictions by themselves, exactly soluble algebraic models can also serve as a useful testground for more sophisticated microscopic theories, in which heavy numerical work is inevitable.

An algebraic approach to the structure of metal clusters 3, 4 has been introduced recently [5, 6, 7, 8, 9, taking advantage of the three-dimensional $q$-deformed harmonic oscillator (3D $q-\mathrm{HO})$ [10, which is constructed using the techniques of quantum algebras (quantum groups) see 11 and references therein]. The symmetry of this oscillator is $\mathrm{u}_{q}(3) \supset \mathrm{so}_{q}(3)$ [12, 13, 14, 15, 16, while its derivation involves use of irreducible tensor operators under $\mathrm{So}_{q}(3)$ [15, 17, 18]. In terms of the 3D $q-\mathrm{HO}$ a good description of the magic numbers of alkali clusters (up to 1500 atoms, which is the limit of validity for theories based on the filling of electronic shells [19, 20]), as well as of Al clusters, has been obtained [5]. In addition, it has been proved that supershells occur naturally in this model [6, 7], which is characterized by only one parameter (the deformation parameter $q=e^{\tau}$, with $\tau$ being real) in addition to the overall scale.

An alternative approach to shell structure coming from mathematical physics has been introduced long before the discovery of metal clusters [21] by Balian and Bloch [22, in the framework of the theory of periodic orbits and classical quantization conditions 23. In this approach the valence electrons of the metal cluster are supposed to move in a spherical cavity (representing the effects of the mean field). Supershells then occur from the superposition of closed classical stationary orbits 23] of the electrons, the superposition 
of triangular and square orbits being the simplest example 24. The theory of Balian and Bloch leads to very specific predictions about the behaviour of magic numbers, which can serve as a test for alternative approaches. For example, the plot of $N_{i}^{1 / 3}$ vs. $i$, where $N_{i}$ are the magic numbers of clusters of a specific alkali metal and $i$ their index $(i=1,2,3$, ... ), should be a straight line with a slope of 0.61 [4, 24 .

In this paper we confront the results provided by the 3D $q$-HO to the restrictions imposed by the theory of Balian and Bloch. It turns out that agreement between the predictions of the two theories can be established by introducing in the Hamiltonian of the 3D $q-\mathrm{HO}$ an additional term, which is characterized by the same symmetry as the original Hamiltonian. Furthermore, it can be seen that this additional term occurs naturally through a variational procedure, analogous to the one used for obtaining the Morse oscillator [25] from the usual harmonic oscillator.

In Section 2 of this paper a brief account of the 3D $q-\mathrm{HO}$ is given, while in Section 3 the application of the periodic orbit theory of Balian and Bloch to metal clusters is briefly described. The predictions of the 3D $q-\mathrm{HO}$ are compared to the restrictions imposed by the theory of Balian and Bloch in Section 4, while in Section 5 a modified Hamiltonian for the $3 \mathrm{D} q-\mathrm{HO}$ is introduced, allowing for full agreement with the theory of Balian and Bloch. Numerical details concerning the modified 3D $q$-HO Hamiltonian are given in Section 6, together with a study of supershells in the framework of this Hamiltonian. In Section 7 a variational method leading from the usual harmonic oscillator to the Morse oscillator is introduced, while in Section 8 this method is applied for deriving the modified Hamiltonian introduced in Section 5 from the original 3D $q$-HO Hamiltonian. Finally in Section 9 a discussion of the present results and plans for future work are given.

\section{The 3-dimensional $q$-deformed harmonic oscillator $(3 \mathrm{D} q-\mathrm{HO})$}

The space of the 3-dimensional $q$-deformed harmonic oscillator consists of the completely symmetric irreducible representations of the quantum algebra $\mathrm{u}_{q}(3)$ [12, 13, 14. In this space a deformed angular momentum algebra, $\mathrm{so}_{q}(3)$, can be defined [10. The Hamiltonian of the 3D $q-\mathrm{HO}$ is defined so that it satisfies the following requirements:

a) It is an $\mathrm{so}_{q}(3)$ scalar, i.e. the energy is simultaneously measurable with the $q$-deformed angular momentum related to the algebra $\mathrm{SO}_{q}(3)$ and its $z$-projection.

b) It conserves the number of bosons, in terms of which the quantum algebras $\mathrm{u}_{q}(3)$ and $\mathrm{SO}_{q}(3)$ are realized.

c) In the limit $q \rightarrow 1$ it is in agreement with the Hamiltonian of the usual 3-dimensional harmonic oscillator.

It has been proved [10] that a Hamiltonian of the 3D q-HO satisfying the above requirements takes the form

$$
H_{q}=\hbar \omega_{0}\left\{[N] q^{N+1}-\frac{q\left(q-q^{-1}\right)}{[2]} C_{q}^{(2)}\right\},
$$

where $N$ is the number operator and $C_{q}^{(2)}$ is the second order Casimir operator of the algebra $\mathrm{SO}_{q}(3)$, while

$$
[x]=\frac{q^{x}-q^{-x}}{q-q^{-1}}
$$

is the definition of $q$-numbers and $q$-operators. In the framework of quantum algebras in general, the deformation parameter $q$ can either be real $\left(q=e^{\tau}\right.$, with $\tau$ being real $)$ or a 
phase factor $\left(q=e^{i \tau}\right.$, with $\tau$ being real). In the present case, however, the parameter $q$ is restricted to obtain real values only, since this is required in the derivation leading to the Hamiltonian of Eq. (1). (See the comment following Eq. (9) in Ref. [16] for more details.)

The energy eigenvalues of the 3D $q-\mathrm{HO}$ are given by [10]

$$
E_{q}(n, l)=\hbar \omega_{0}\left\{[n] q^{n+1}-\frac{q\left(q-q^{-1}\right)}{[2]}[l][l+1]\right\}=\hbar \omega_{0} e_{q}(n, l),
$$

where $n$ is the number of vibrational quanta and $l$ is the eigenvalue of the angular momentum, obtaining the values $l=n, n-2, \ldots, 0$ or 1 , and

$$
e_{q}(n, l)=[n] q^{n+1}-\frac{q\left(q-q^{-1}\right)}{[2]}[l][l+1] .
$$

In the limit of $q \rightarrow 1$ one obtains $\lim _{q \rightarrow 1} E_{q}(n, l)=\hbar \omega_{0} n$, which coincides with the classical result.

For small values of the deformation parameter $\tau$ (where $q=e^{\tau}$ ) one can expand Eq. (3) in powers of $\tau$ obtaining [10]

$$
\begin{gathered}
E_{q}(n, l)=\hbar \omega_{0} n-\hbar \omega_{0} \tau\{l(l+1)-n(n+1)\} \\
-\hbar \omega_{0} \tau^{2}\left\{l(l+1)-\frac{1}{3} n(n+1)(2 n+1)\right\}+\mathcal{O}\left(\tau^{3}\right) .
\end{gathered}
$$

The last expression to leading order bears great similarity to the modified harmonic oscillator suggested by Nilsson [26, 27] (with the spin-orbit term omitted). Comparisons between the predictions of the 3D $q-\mathrm{HO}$ and Nilsson's modified oscillator for the magic numbers and supershells of metal clusters have been given in 5, 6, 7. One of the main differences between the two models is that the term $\tau n(n+1)$ in the eigenvalues of the $3 \mathrm{D} q-\mathrm{HO}$ given in Eq. (5) is occuring as a consequence of the overall symmetry, while the corresponding term $\mu^{\prime} n(n+3) / 2$ in the eigenvalues of Nilsson's modified oscillator (see, for example, Eq. (3) of Ref. [1]) is put in "by hand". It should also be noticed that the use of $q$-deformations does not mean that an additional parameter is introduced in the theory. In both the 3D $q$-HO and Nilsson's modified oscillator only one parameter appears (in addition to the overall scale). In the 3D $q-\mathrm{HO}$ case this is the deformation parameter $\tau$, while in Nilsson's modified oscillator it is the parameter $\mu^{\prime}$ (see, for example, Eq. (3) of Ref. [7]).

\section{The theory of Balian and Bloch}

The theory of Balian and Bloch [22] is an example of periodic orbit theory [23, developed as a semiclassical bridge between quantum mechanics and classical mechanics. In the approach of Balian and Bloch, shell effects in metal clusters are studied by considering the valence electrons moving on straight lines within a smooth sphere and being reflected on the inner surface of the sphere. Periodic orbits in this case correspond to various polygons with three or more corners, the triangular and the square orbits being the simplest examples 4, 24.

In the theory of Balian and Bloch a cluster of $N$ alkali atoms can be studied 24 by considering the $N$ valence electrons moving in a sphere with radius

$$
R=r_{S} N^{1 / 3}
$$


where $r_{S}$ is the Wigner-Seitz radius. The lengths of the triangular and the square orbits are then respectively

$$
\begin{aligned}
& L_{3}=3 \sqrt{3} r_{S} N^{1 / 3}, \\
& L_{4}=4 \sqrt{2} r_{S} N^{1 / 3} .
\end{aligned}
$$

Assuming that electrons move with the Fermi velocity $v_{F}$, the beating pattern created by the superposition of the two orbits leads to supershell structure. It turns out that the magic numbers (shell closures) $N_{i}$ are related to the index $i$ counting their number $(i=1,2,3$, ...) by 24 ]

$$
N_{i}^{1 / 3}=\frac{h}{m v_{F} r_{S}} \quad \frac{2}{3 \sqrt{3}+4 \sqrt{2}} \quad i=0.605 i,
$$

where $m$ is the electron mass and the fact that $v_{F} r_{S}$ is constant for all metals has been used. Therefore the plot of $N_{i}^{1 / 3}$ vs. $i$ should be a straight line with a slope of 0.61 . Furthermore, a phase shift of a half unit of the index $i$ should occur in the node region of the beat pattern, i.e., when passing from a supershell to the next one [4, 24. In addition, within each supershell the shell closures should appear at equidistant positions (i.e., they should exhibit a periodicity) when plotted vs. $N^{1 / 3}$ [4, where $N$ is the number of particles (valence electrons in the present case).

Comparisons to experimental sets of magic numbers have shown 4 that a slope of 0.61 is obtained in the cases of $\mathrm{Na}$ and Li clusters, revealing that the triangular and square orbits represent a good approximation in these cases. For Al clusters, though, a slope of 0.32 has been obtained 4, indicating that in this case, if the theory is applicable, more complicated orbits enter [28].

\section{Comparing the predictions of the $3 \mathrm{D} q-\mathrm{HO}$ and of the Balian-Bloch theory}

As we have seen in the previous section, a hallmark of the theory of Balian and Bloch is that the plot of $N_{i}^{1 / 3}$ vs. $i$, where $N_{i}$ are the magic numbers and $i$ the index counting them $(i=1,2,3, \ldots)$, should be a straight line having a slope of 0.61 in the case of alkali metals, while in the case of $\mathrm{Al}$ clusters the slope should be 0.32 4.

In order to compare the predictions of the $3 \mathrm{D} q-\mathrm{HO}$ to the restrictions imposed by the theory of Balian and Bloch, we plot in Fig. 1(a) (line labelled by $\epsilon=0.0$ ) the magic numbers obtained from the 3D $q$-HO for $\tau=0.038$, the parameter value found appropriate in Ref. 5 for reproducing the magic numbers of alkali clusters (up to 1500 atoms, which is the limit of validity of theories based on the filling of electronic shells [19, 20]). Numbers considered as magic, listed in Table 1, correspond to energy gaps larger than $\delta=0.38$, as in Ref. [5], with $\hbar \omega_{0}=1$. In the same figure, a straight line with a slope of 0.61 appears. It is clear that the 3D $q$-HO magic numbers follow the straight line up to $i=14$ quite well, while beyond this point the predictions of the $3 \mathrm{D} q-\mathrm{HO}$ are clearly lower than the straight line, indicating that "too many" magic numbers are produced by the model in this region.

The same conclusion is arrived at by looking at Fig. 1(b), where the predictions of the 3D $q$-HO for $\tau=0.050$, the parameter value found in Ref. 5] appropriate for reproducing the magic numbers of $\mathrm{Al}$ clusters, are reported (line labelled by $\epsilon=0.0$ ). Again magic numbers, listed in Table 1, correspond to energy gaps larger than $\delta=0.38$ (with $\left.\hbar \omega_{0}=1\right)$. Small magic numbers (below 186, i.e., below $i=9$ ) are not shown, since it is known that small magic numbers in Al clusters cannot be explained by models based on the filling of electronic shells, because of the symmetry breaking caused by the ionic lattice [29], while for large magic numbers this problem does not exist. In the plot a straight line with a slope 
of 0.32 , which is expected to be appropriate for $\mathrm{Al}$ clusters 4, as mentioned in the previous section, is also seen. It is clear that the predictions of the 3D $q$-HO follow a line parallel to the one with slope 0.32 roughly up to $i=23$, while beyond this point the slope is gradually reduced, indicating that the model predicts "too many" magic numbers.

\section{A modified Hamiltonian for the 3D $q-\mathrm{HO}$}

The discrepancy between the 3D $q$-HO and the theory of Balian and Bloch can be lifted by considering the Hamiltonian

$$
H_{q}^{\prime}=H_{q}-\epsilon H_{q}^{2}
$$

with eigenvalues

$$
E_{q}^{\prime}(n, l)=E_{q}(n, l)-\epsilon E_{q}^{2}(n, l),
$$

where $\epsilon$ is a small real positive constant. Justification for this choice will be given in Sections 7 and 8 through a variational procedure. For the moment the following comments suffice:

a) It is clear that $H_{q}^{\prime}$ is a function of $H_{q}$, which is by construction an $\operatorname{so}_{q}(3)$ scalar, as mentioned in Section 2. Therefore $H_{q}^{\prime}$ is also an $\mathrm{so}_{q}(3)$ scalar.

b) The energy eigenvalues of the new Hamiltonian can be written in the form

$$
E_{q}^{\prime}(n, l)=\hbar \omega_{0}\left(1-\epsilon \hbar \omega_{0} e_{q}(n, l)\right) e_{q}(n, l)=\hbar \omega(n, l) e_{q}(n, l),
$$

where

$$
\omega(n, l)=\omega_{0}\left(1-\epsilon \hbar \omega_{0} e_{q}(n, l)\right)
$$

is a variable frequency, depending on the quantum numbers $n, l$, and on the small parameter $\epsilon$. We shall call this oscillator the Variable Frequency Oscillator (VFO) corresponding to the 3D $q$-HO, a term for which justification will be provided in Sections 7 and 8.

The magic numbers provided by the VFO for a few appropriate values of $\epsilon$ in the case of $\tau=0.038$, which is relevant for alkali clusters [5], are shown in Fig. 1(a) and listed in Table 1. Details of the calculation will be given in Section 6. Once more magic numbers are separated by gaps larger than $\delta=0.38$, while $\hbar \omega_{0}=1$. It is clear that the predictions of the VFO roughly follow the straight line with a slope of 0.61 even for large values of $i$, thus overcoming the difficulties faced by the 3D $q$-HO.

A similar picture is obtained for $\tau=0.050$ (and $\delta=0.38$, with $\hbar \omega_{0}=1$ ), which is appropriate for Al clusters [5]. For a few appropriate values of $\epsilon$, shown in Fig. 1(b) and listed in Table 1, the predictions of the VFO roughly follow a straight line with a slope of 0.32 , as they should, according to the previous section.

We therefore conclude that the addition of the second term in the Hamiltonian of the $3 \mathrm{D} q$-HO, leading to the VFO, makes the predictions of the 3D $q$-HO compatible with the predictions of the theory of Balian and Bloch.

\section{$6 \quad$ Numerical details}

In this section the calculations leading to the results reported in Section 5 will be described. Throughout this paper we put $\hbar \omega_{0}=1$ for simplicity.

An important difference between the 3D q-HO and the VFO of Eq. (10) lies in the way truncations of the spectrum are made. The following comments apply:

a) In the 3D $q$-HO the level with $l=n$ always lies lowest in energy within each shell, the level with $l=n-2$ lies immediately above it, and so on. Therefore stopping the level scheme at the $l=n$ level of a given shell and taking into account all levels with lower $n$ 
(i.e., all levels of the shells lying below the given one), one makes sure that all levels up to the given level have been included [7.

b) In the case of the VFO of Eq. (10) the following picture occurs: For a given (small) value of $\epsilon$ the first several shells exhibit the same behaviour as in the case of the 3D $q$-HO, i.e., the level with $l=n$ lies lowest in energy within each shell, the level with $l=n-2$ lies immediately above it, and so on. As the shell number is increasing, however, an inversion of the order of the levels occurs, with the levels with $l=0$ (for $n$ being even) or $l=1$ (for $n$ being odd) lying lowest in energy within the shell. It is then clear that beyond this inversion point truncation at a given shell should be made at the level with $l=0$ (for $n$ being even) or at $l=1$ (for $n$ being odd).

The magic numbers obtained for the values of $\tau$ and $\epsilon$ used in the previous section are given in Table 1 . In each case the maximum value of $n, n_{\max }$, included in the calculation is indicated. Care has been taken that no inversion of the order of the levels, of the type described in comment b) above, occurs for the values of $n$ included in the calculation. Therefore in all cases truncation is made at the level with $n=n_{\max }$ and $l=n_{\max }$. The total number of levels up to the truncation point, $N_{\max }$, is also shown in Table 1 . The following remarks are now in place:

a) In the cases considered here and up to the truncation point, for a given value of $\tau$ the order of the levels is not modified as $\epsilon$ is changed. The only modification occuring is that the spectrum gets "squeezed" as $\epsilon$ increases.

b) As a result of a), the magic numbers reported in Table 1 present the following feature. For each value of $\tau$ and for $\epsilon=0.0$ the largest number of magic numbers appears. As $\epsilon$ increases, some of the magic numbers cease to be magic any more, since the "squeezing" of the spectrum brings the levels closer to each other. For each value of $\epsilon$ the magic numbers occuring are a subset of the magic numbers occuring for lower values of $\epsilon$ (with the same $\tau)$. No new magic numbers appear, within the limits considered here, as $\epsilon$ increases.

It is interesting to examine at this point what the influence of the additional term to the appearance of supershells is. For this purpose we are going to use the procedure employed by Nishioka et al. [30, 31. For a given number of particles $N$ the single particle energies $E_{j}(n, l)$ of the $N$ occupied states are summed up

$$
E(N)=\sum_{j=1}^{N} E_{j}(n, l) .
$$

This sum is then divided into two parts: A smooth average part $E_{a v}$ and a shell part $E_{\text {shell }}$, which will exhibit the supeshell structure

$$
E(N)=E_{a v}(N)+E_{\text {shell }}(N) .
$$

For the average part of the total energy a Liquid Drop Model expansion is used [7]

$$
E_{a v}(N)=a_{1} N^{1 / 3}+a_{2} N^{2 / 3}+a_{3} N+a_{4} N^{4 / 3}+a_{5} N^{5 / 3}+a_{6} N^{2} .
$$

The parameters of the fits occuring in the cases considered here are shown in Table 2, together with the number of levels, $N_{\max }$, included in the fit and the rms deviation $\sigma$. We remark that for a given value of $\tau$ the parameters change smoothly for the nonzero values of $\epsilon$, while the case with $\epsilon=0.0$ is characterized by quite different values of the parameters, but also by a higher rms deviation $\sigma$. The addition of the second term in Eq. (10) improves the agreement of the average part of the total energy to the Liquid Drop Model expansion, thus resulting in lower rms deviations $\sigma$.

The procedure of the calculation was as follows: First the summations described by Eq. (14), resulting in the total energy $E(N)$ for each particle number $N$, have been performed. 
Subsequently, in order to reduce the size of the calculation approximately by a factor of 10 , the average $E(N)$ was calculated every 11 points (i.e., for $N=6,17,28, \ldots$ ) up to the cutoff point which is reported in Table 2 as $N_{\max }$. These averaged values of $E(N)$ were subsequently fitted by the expansion of Eq. (16), resulting in the determination of $E_{a v}(N)$ at these points. Finally $E_{\text {shell }}(N)$ has been obtained at these points as the difference $E(N)-E_{a v}(N)$ and plotted in Figs. 2 and 3.

The shell energy, $E_{\text {shell }}$, is plotted vs. the particle number, $N$, for $\tau=0.038$, which is appropriate for alkali clusters [5], in Fig. 2. In Fig. 2(a) the predictions of the original 3D $q-\mathrm{HO}$ (with $\epsilon=0$ ) are shown, while in Fig. 2(b) the results of the VFO with $\epsilon=0.006$ are depicted. The VFO with $\epsilon=0.007,0.008$ gives results which look almost identical with Fig. 2(b) and therefore are not shown for brevity. The similarities between Figs. 2(a) and 2 (b) are clear. Not only the supershell appears in both cases around $N=1000$, as it is expected for Na clusters [30, 31, but in addition even the maxima and minima of the shell energy appear at the same particle numbers and have roughly the same magnitude. Even the local maxima and minima present striking similarities. These results corroborate the remarks made above, namely that the addition of the second term to the Hamiltonian of Eq. (10) does not influence the order of the energy levels, the main effect of the second term being the gradual "squeezing" of the spectrum as energy increases. Of course this conclusion is valid only within the region of particle numbers studied and for small values of $\epsilon$, like the ones used here.

Similar results are obtained in Fig. 3 for the case of $\tau=0.050$, which is appropriate for Al clusters [5]. Besides the $\epsilon=0$ case, shown in Fig. 3(a), the results corresponding to $\epsilon=0.0050$ are shown in Fig. 3(b), since the cases with $\epsilon=0.0053$, 0.0055 provide results almost identical with the ones shown in Fig. 3(b). In all cases there is some evidence for a supershell below $N=1000$, although its appearance is not as clear as in the case of Fig. 2. The appearance of a supershell in this region is in agreement with the results of more sophisticated calculations, as, for example, spherical jellium model predictions in Local Density Approximation [32, but it is not in good agreement with experiment, where no evidence for supershell in Al clusters exists in this region [33, 34. An advantage of the VFO in comparison to earlier calculations 32 is that at least it can reproduce the slope of 0.32 in Fig. 1(b), something which is not occuring in spherical jellium model calculations, although it occurs experimentally [4, 33, 34].

In the theory of Balian and Bloch, as mentioned above, a phase shift by a half unit of the running index $i$ should be observed in the plot of the magic numbers $N_{i}^{1 / 3}$ vs. $i$ when passing from a supershell to the next [4, 24]. In Fig. 1(a) such a shift is seen quite clearly around $N_{i}=1000$ in the cases of $\epsilon=0.006,0.007,0.008$, while no clear shift of this type is seen in Fig. 1(b).

Furthermore, the theory of Balian and Bloch, as mentioned above, predicts that within each supershell the minima of the shell energy, $E_{\text {shell }}$, should appear at equidistant positions (i.e. they should exhibit a periodicity) when plotted vs. $N^{1 / 3}$. The change in the periodicity when passing from the first supershell to the second one is clear in Figs. 2 and 3 (although in these cases, for reasons of clarity, $E_{\text {shell }}$ is plotted vs. $N$ and not vs. $N^{1 / 3}$ ). The fact that the predictions of the VFO corresponding to the $3 \mathrm{D} q-\mathrm{HO}$ approximately exhibit the right periodicity features does not come as a surprise, since the $3 \mathrm{D} q$-HO is known to show this feature [6, 7, while, as we have seen above, the addition of the second term in Eq. (10) does not influence the position of the minima.

In conclusion, the VFO corresponding to the $3 \mathrm{D} q-\mathrm{HO}$ is able to reproduce the right slope in the $N_{i}^{1 / 3}$ vs. $i$ plot in both the alkali and Al clusters. In addition it predicts correctly the first supershell in alkali clusters, while in Al clusters its prediction for a supershell is in rough agreement with results of spherical jellium models but not with experiment. 


\section{A variational method}

In nuclear physics it is well known that nuclear spectra can be described very accurately in terms of the Variable Moment of Inertia (VMI) model [35]. In this model the energy levels are given by

$$
E(J)=\frac{J(J+1)}{2 \Theta(J)}+\frac{1}{2} C\{\Theta(J)-\Theta(0)\}^{2},
$$

where $J$ is the angular momentum and $\Theta(J)$ is the moment of inertia, which is supposed to be a function of the angular momentum. $C$ and $\Theta(0)$ are free parameters, the latter representing the ground state moment of inertia. It is clear that the VMI formula is a generalization of the rigid rotator formula

$$
E(J)=\frac{J(J+1)}{2 \Theta},
$$

in which the moment of inertia is assumed to be constant. The rigid rotator formula is known to fail beyond the first few levels of a rotational nucleus, since the experimental levels appear "squeezed" in comparison to the rigid rotator predictions. This difficulty is overcome in the framework of the VMI model by determining the moment of inertia for each value of the angular momentum $J$ through a minimization of the energy with respect to the moment of inertia for given angular momentum

$$
\left.\frac{\partial E(J)}{\partial \Theta(J)}\right|_{J}=0
$$

This variational condition leads to a cubic equation for $\Theta(J)$, which turns out to have only one real solution [35, corresponding to the appropriate value of the moment of inertia for the given value of the angular momentum. The second term in Eq. (17) is justified by the well known fact that many perturbing potentials near their origin can be approximated by a harmonic oscillator potential.

Following the same reasoning, it is interesting to examine what happens to the usual harmonic oscillator if, by analogy, one allows the angular frequency to be a function of the quantum number $n$. The energy will then read

$$
E(n)=\hbar \omega(n)\left(n+\frac{1}{2}\right)+\frac{1}{2} C\{\omega(n)-\omega(0)\}^{2},
$$

where $C$ and $\omega(0)$ are free parameters, the latter corresponding to the ground state angular frequency. The variational principle in this case should correspond to the minimization of the energy with respect to the angular frequency for constant value of the quantum number $n$

$$
\left.\frac{\partial E(n)}{\partial \omega(n)}\right|_{n}=0 \text {. }
$$

It is clear that this condition leads to

$$
\hbar\left(n+\frac{1}{2}\right)+C\{\omega(n)-\omega(0)\}=0 \Rightarrow \omega(n)=\omega(0)-\frac{\hbar}{C}\left(n+\frac{1}{2}\right) .
$$

Substituting this result in Eq. (20) we obtain

$$
E(n)=\hbar \omega(0)\left(n+\frac{1}{2}\right)-\frac{1}{2} \frac{\hbar^{2}}{C}\left(n+\frac{1}{2}\right)^{2},
$$


which is reminiscent of the spectrum of the Morse potential [25].

Indeed, solving the Schrödinger equation for the Morse potential [36, 37, 38,

$$
V(x)=D\left(1-e^{-\alpha x}\right)^{2},
$$

one obtains the energy spectrum

$$
E(n)=\hbar \omega\left\{\left(n+\frac{1}{2}\right)-x_{e}\left(n+\frac{1}{2}\right)^{2}\right\}
$$

where

$$
x_{e}=\frac{1}{2} \frac{\hbar \alpha}{\sqrt{2 m D}}
$$

and

$$
\omega=\alpha \sqrt{\frac{2 D}{m}} .
$$

We therefore conclude that by allowing the angular frequency of the simple harmonic oscillator to vary with the quantum number $n$, we obtain the spectrum of the Morse oscillator. Again the second term in Eq. (20) is in agreement to the fact that most perturbing potentials near their origin can be approximated by the harmonic oscillator potential. We shall refer to the oscillator of Eq. (20) as the Variable Frequency Oscillator (VFO).

\section{Derivation of the modified Hamiltonian for the 3D $q-\mathrm{HO}$ through a variational method}

The idea leading to the VFO of the previous section can be appropriately generalized in the case of the 3D $q$-HO. In this case we consider the energy expression

$$
\begin{gathered}
E_{q}^{\prime}(n, l)=\hbar \omega(n, l)\left\{[n] q^{n+1}-\frac{q\left(q-q^{-1}\right)}{[2]}[l][l+1]\right\}+\frac{1}{2} C\{\omega(n, l)-\omega(0,0)\}^{2} \\
=\hbar \omega(n, l) e_{q}(n, l)+\frac{1}{2} C\{\omega(n, l)-\omega(0,0)\}^{2}
\end{gathered}
$$

where the angular frequency $\omega(n, l)$ depends on the quantum numbers $n$ and $l$, while $C$ and $\omega(0,0)$ are real positive constants, the latter corresponding to the ground state angular frequency, since the ground state of the 3D $q-\mathrm{HO}$ is characterized by $n=0$ and $l=0$. It is thus clear that $\omega(0,0)$ corresponds to $\omega_{0}$ appearing in Eq. (3), i.e., $\omega(0,0) \equiv \omega_{0}$. The variational condition in the present case should read

$$
\left.\frac{\partial E_{q}^{\prime}(n, l)}{\partial \omega(n, l)}\right|_{n, l}=0 .
$$

In other words, the energy is minimized with respect to the angular frequency for constant values of the quantum numbers $n$ and $l$. The variational condition leads to the equation

$$
\hbar e_{q}(n, l)+C\{\omega(n, l)-\omega(0,0)\}=0 \Rightarrow \omega(n, l)=\omega(0,0)-\frac{\hbar}{C} e_{q}(n, l)
$$




$$
=\omega(0,0)-\frac{\hbar}{C}\left\{[n] q^{n+1}-\frac{q\left(q-q^{-1}\right)}{[2]}[l][l+1]\right\} .
$$

Substituting this result in Eq. (28) one then obtains

$$
E_{q}^{\prime}(n, l)=\hbar \omega(0,0) e_{q}(n, l)-\frac{1}{2} \frac{\hbar^{2}}{C} e_{q}^{2}(n, l)
$$

which is the same as Eq. (11), with

$$
\epsilon=\frac{1}{2 C \omega_{0}^{2}}
$$

since $\omega_{0} \equiv \omega(0,0)$, as mentioned above.

\section{Discussion}

In this paper we have attempted a comparison of the predictions for the shell structure of metal clusters of the 3D q-HO model to the ones of the periodic orbit theory of Balian and Bloch. It turns out that the predictions of the $3 \mathrm{D} q-\mathrm{HO}$ for the magic numbers of metal clusters can be made compatible with the predictions of the theory of Balian and Bloch by adding to the 3D $q-\mathrm{HO}$ a symmetry-preserving correction term reminiscent of the anharmonicity term in the spectrum of the Morse potential, while this addition does not influence the predictions for the supershells. This extended expression for the 3D $q$-HO can be justified through a variational method, similar to the one used in the Variable Moment of Inertia (VMI) model of nuclear physics, leading to the concept of the Variable Frequency Oscillator (VFO), which gives promise of wider applicability.

\section{Acknowledgements}

One of the authors (PPR) acknowledges support from the Bulgarian Ministry of Science and Education under contracts $\Phi-415$ and $\Phi-547$.

\section{References}

[1] F. Iachello and A. Arima, The Interacting Boson Model (Cambridge University Press, Cambridge, 1987).

[2] F. Iachello and R. D. Levine, Algebraic Theory of Molecules (Oxford University Press, Oxford, 1995).

[3] W. A. de Heer, Rev. Mod. Phys. 65, 611-676 (1993).

[4] M. Brack, Rev. Mod. Phys. 65, 677-732 (1993).

[5] D. Bonatsos, N. Karoussos, D. Lenis, P. P. Raychev, R. P. Roussev and P. A. Terziev, Phys. Rev. A 62, 013203 [13 pages] (2000).

[6] D. Bonatsos, D. Lenis, P. P. Raychev and P. A. Terziev, Int. J. Quant. Chem. 89, 299-312 (2002). 
[7] D. Bonatsos, D. Lenis, P. P. Raychev and P. A. Terziev, Phys. Rev. A 65, 033203 [12 pages] (2002).

[8] A. I. Kuleff, J. Maruani and P. P. Raychev, Adv. Quant. Chem. 40, 279-304 (2001).

[9] D. Bonatsos, A. I. Kuleff, J. Maruani, P. P. Raychev and P. A. Terziev, Int. J. Quant. Chem. 89, 377-388 (2002).

[10] P. P. Raychev, R. P. Roussev, N. Lo Iudice and P. A. Terziev, J. Phys. G: Nucl. Part. Phys. 24, 1931-1943 (1998).

[11] D. Bonatsos and C. Daskaloyannis, Prog. Part. Nucl. Phys. 43, 537-618 (1999).

[12] Yu. F. Smirnov, V. N. Tolstoy and Yu. I. Kharitonov, Yad. Fiz. 54, 721-736 (1991) [Sov. J. Nucl. Phys. 54, 437-445 (1991)].

[13] J. Van der Jeugt, J. Phys. A: Math. Gen. 25, L213-L218 (1992).

[14] J. Van der Jeugt, J. Math. Phys. 34, 1799-1806 (1993).

[15] C. Quesne, Phys. Lett. B 304, 81-88 (1993).

[16] P. P. Raychev, R. P. Roussev, P. A. Terziev, D. Bonatsos and N. Lo Iudice, J. Phys. A: Math. Gen. 29, 6939-6949 (1996).

[17] Yu. F. Smirnov, V. N. Tolstoy and Yu. I. Kharitonov, Yad. Fiz. 53, 959-980 (1991) [Sov. J. Nucl. Phys. 53, 593-605 (1991)].

[18] Yu. F. Smirnov, V. N. Tolstoy and Yu. I. Kharitonov, Yad. Fiz. 56, 223-244 (1993) [Phys. At. Nucl. 56, 690-700 (1993)].

[19] T. P. Martin, T. Bergmann, H. Göhlich and T. Lange, Chem. Phys. Lett. 172, 209-213 (1990).

[20] K. Clemenger, Phys. Rev. B 44, 12991-13001 (1991).

[21] W. D. Knight, K. Clemenger, W. A. de Heer, W. A. Saunders, M. Y. Chou and M. L. Cohen, Phys. Rev. Lett. 52, 2141-2143 (1984).

[22] R. Balian and C. Bloch, Ann. Phys. (N.Y.) 69, 76-160 (1972).

[23] M. C. Gutzwiller, J. Math. Phys. 12, 343-358 (1971).

[24] C. Bréchignac, Ph. Cahuzac, F. Carlier, M. de Frutos and J. Ph. Roux, Phys. Rev. B 47, 2271-2277 (1993).

[25] P. Morse, Phys. Rev. 34, 57-64 (1929).

[26] S. G. Nilsson, Mat. Fys. Medd. K. Dan. Vidensk. Selsk. 29, 16 (1955).

[27] S. G. Nilsson and I. Ragnarsson, Shapes and Shells in Nuclear Structure (Cambridge University Press, Cambridge, 1995).

[28] J. Lermé, M. Pellarin, J. L. Vialle, B. Baguenard and M. Broyer, Phys. Rev. Lett. 68, 2818-2821 (1992). 
[29] J. L. Persson, R. L. Whetten, H. P. Cheng and R. S. Berry, Chem. Phys. Lett. 186, 215-222 (1991).

[30] H. Nishioka, K. Hansen and B. R. Mottelson, Phys. Rev. B 42, 9377-9386 (1990).

[31] H. Nishioka, Z. Phys. D 19, 19-23 (1991).

[32] O. Genzken, M. Brack, E. Chabanat and J. Meyer, Ber. Bunsenges. Phys. Chem. 96, 1217-1220 (1992).

[33] M. Pellarin, J. Lermé, B. Baguenard, M. Broyer and J. L. Vialle, Ber. Bunsenges. Phys. Chem. 96, 1212-1215 (1992).

[34] M. Pellarin, B. Baguenard, M. Broyer, J. Lermé and J. L. Vialle, J. Chem. Phys. 98, 944-950 (1993).

[35] M. A. J. Mariscotti, G. Scharff-Goldhaber and B. Buck, Phys. Rev. 178, 1864-1887 (1969).

[36] S. Flügge, Practical Quantum Mechanics (Springer, Berlin, 1974).

[37] I. L. Cooper, Chem. Phys. 112, 67-75 (1987).

[38] I. L. Cooper, J. Phys. A: Math. Gen. 25, 1671-1683 (1992).

\section{Figure captions}

Fig. 1 Cubic roots of the magic numbers $N_{i}$ plotted vs. the running index $i$ counting them. The magic numbers are the ones listed in Table 1. (a) $\tau=0.038$, (b) $\tau=0.050$.

Fig. 2 Shell part $\left(E_{\text {shell }}\right)$ of the total energy [in units of $\hbar \omega_{0}$, see Eqs. (31) and (11)] vs. the number of particles $N$, in the case of $\tau=0.038$, (a) for the 3D $q-\mathrm{HO}$, (b) for a corresponding VFO. The values of the dimensionless parameters $\tau$ and $\epsilon$ are listed in Table 2 , together with the details of the calculation. See Section 6 for further discussion.

Fig. 3 Same as Fig. 2, but for $\tau=0.050$. 
Table 1: Magic numbers (corresponding to gaps larger than $\delta=0.38$, with $\hbar \omega_{0}=1$ ) produced by the 3D $q$-HO [Eq. (31)] (cases with $\epsilon=0.0$ ) and the corresponding VFO [Eq. (111)] for different values of the parameters $\tau$ and $\epsilon . n_{\max }$ is the maximum value of the quantum number $n$ included in the calculation, while the highest level taken into account is the one with $n=n_{\max }$ and $l=n_{\max }$, corresponding to the reported total number of particles $N_{\max } . i$ is a running index counting the magic numbers. See Section 6 for further discussion.

\begin{tabular}{rrrrrrrrr}
\hline$\tau$ & 0.038 & 0.038 & 0.038 & 0.038 & 0.050 & 0.050 & 0.050 & 0.050 \\
$\epsilon$ & 0.0 & 0.006 & 0.007 & 0.008 & 0.0 & 0.0050 & 0.0053 & 0.0055 \\
$n_{\max }$ & 26 & 26 & 26 & 25 & 26 & 26 & 25 & 25 \\
$N_{\max }$ & 4658 & 4658 & 4658 & 4154 & 4778 & 4778 & 4258 & 4258 \\
\hline$i$ & & & & & & & & \\
1 & 2 & 2 & 2 & 2 & 2 & 2 & 2 & 2 \\
2 & 8 & 8 & 8 & 8 & 8 & 8 & 8 & 8 \\
3 & 20 & 20 & 20 & 20 & 20 & 20 & 20 & 20 \\
4 & 34 & 34 & 40 & 40 & 34 & 34 & 34 & 34 \\
5 & 40 & 40 & 58 & 58 & 40 & 40 & 40 & 40 \\
6 & 58 & 58 & 92 & 92 & 58 & 58 & 58 & 58 \\
7 & 92 & 92 & 138 & 138 & 92 & 92 & 92 & 92 \\
8 & 138 & 138 & 198 & 198 & 138 & 138 & 138 & 138 \\
9 & 198 & 198 & 254 & 254 & 186 & 186 & 186 & 186 \\
10 & 254 & 254 & 338 & 338 & 254 & 254 & 254 & 254 \\
11 & 268 & 338 & 440 & 440 & 338 & 338 & 338 & 338 \\
12 & 338 & 440 & 676 & 676 & 398 & 398 & 398 & 398 \\
13 & 440 & 676 & 832 & 832 & 440 & 440 & 440 & 440 \\
14 & 556 & 832 & 912 & 912 & 486 & 542 & 542 & 542 \\
15 & 562 & 912 & 1012 & 1012 & 542 & 612 & 612 & 612 \\
16 & 676 & 1012 & 1100 & 1100 & 612 & 676 & 676 & 676 \\
17 & 694 & 1100 & 1206 & 1206 & 676 & 748 & 748 & 748 \\
18 & 832 & 1206 & 1660 & 1660 & 748 & 832 & 832 & 832 \\
19 & 912 & 1660 & 1760 & 1760 & 832 & 912 & 912 & 912 \\
20 & 1012 & 1760 & 2048 & 2048 & 890 & 1006 & 1006 & 1006 \\
21 & 1100 & 2048 & 2368 & 2368 & 912 & 1074 & 1074 & 1074 \\
22 & 1206 & 2368 & 3028 & 3028 & 1006 & 1100 & 1100 & 1100 \\
23 & 1284 & 3028 & 3438 & 3438 & 1074 & 1284 & 1284 & 1284 \\
24 & 1314 & 3438 & 3886 & 3886 & 1100 & 1314 & 1314 & 1410 \\
25 & 1410 & 3886 & 4374 & & 1206 & 1410 & 1410 & 1502 \\
26 & 1502 & 4052 & & & 1284 & 1502 & 1502 & 1760 \\
27 & 1516 & 4374 & & & 1314 & 1516 & 1760 & 2018 \\
28 & 1660 & & & & 1410 & 1760 & 2018 & 2048 \\
29 & 1760 & & & & 1502 & 2018 & 2048 & 2178 \\
30 & 2018 & & & & 1516 & 2048 & 2178 & 2334 \\
\hline & & & & & & & &
\end{tabular}


Table 1: (continued)

\begin{tabular}{|c|c|c|c|c|c|c|c|c|}
\hline $\bar{\tau}$ & 0.038 & 0.038 & 0.038 & 0.038 & 0.050 & 0.050 & 0.050 & 0.050 \\
\hline$\epsilon$ & 0.0 & 0.006 & 0.007 & 0.008 & 0.0 & 0.0050 & 0.0053 & 0.0055 \\
\hline$n_{\max }$ & 26 & 26 & 26 & 25 & 26 & 26 & 25 & 25 \\
\hline$N_{\max }$ & 4658 & 4658 & 4658 & 4154 & 4778 & 4778 & 4258 & 4258 \\
\hline$i$ & & & & & & & & \\
\hline 31 & 2048 & & & & 1614 & 2178 & 2334 & 2368 \\
\hline 32 & 2178 & & & & 1660 & 2334 & 2368 & 2510 \\
\hline 33 & 2334 & & & & 1734 & 2368 & 2510 & 2672 \\
\hline 34 & 2368 & & & & 1760 & 2510 & 2672 & 2722 \\
\hline 35 & 2654 & & & & 1778 & 2672 & 2722 & 3028 \\
\hline 36 & 2672 & & & & 1940 & 2722 & 3028 & 3050 \\
\hline 37 & 2722 & & & & 2018 & 3028 & 3050 & 3112 \\
\hline 38 & 2796 & & & & 2048 & 3050 & 3112 & 3438 \\
\hline 39 & 3028 & & & & 2178 & 3112 & 3438 & 3464 \\
\hline 40 & 3050 & & & & 2334 & 3438 & 3464 & 3886 \\
\hline 41 & 3190 & & & & 2368 & 3464 & 3886 & 3916 \\
\hline 42 & 3404 & & & & 2510 & 3886 & 3916 & \\
\hline 43 & 3438 & & & & 2672 & 3916 & & \\
\hline 44 & 3464 & & & & 2684 & 3988 & & \\
\hline 45 & 3610 & & & & 2722 & 4374 & & \\
\hline 46 & 3848 & & & & 2876 & 4408 & & \\
\hline 47 & 3886 & & & & 3028 & & & \\
\hline 48 & 4052 & & & & 3050 & & & \\
\hline 49 & 4312 & & & & 3112 & & & \\
\hline 50 & 4326 & & & & 3190 & & & \\
\hline 51 & 4374 & & & & 3244 & & & \\
\hline 52 & 4552 & & & & 3438 & & & \\
\hline 53 & & & & & 3464 & & & \\
\hline 54 & & & & & 3528 & & & \\
\hline 55 & & & & & 3622 & & & \\
\hline 56 & & & & & 3680 & & & \\
\hline 57 & & & & & 3886 & & & \\
\hline 58 & & & & & 3916 & & & \\
\hline 59 & & & & & 3988 & & & \\
\hline 60 & & & & & 4088 & & & \\
\hline 61 & & & & & 4156 & & & \\
\hline 62 & & & & & 4374 & & & \\
\hline 63 & & & & & 4408 & & & \\
\hline 64 & & & & & 4462 & & & \\
\hline 65 & & & & & 4488 & & & \\
\hline 66 & & & & & 4578 & & & \\
\hline 67 & & & & & 4596 & & & \\
\hline
\end{tabular}


Table 2: Parameters used for fitting the average part of the total energy [see Eq. (16)] in the case of the 3D $q$-HO and the corresponding VFO for various values of the parameters $\tau$ and $\epsilon$, corresponding to the cases exhibited in Figs. 2 and 3. The parameters are dimensionless, since we have assumed $\hbar \omega_{0}=1$ [see Eqs. (3) and (11)] throughout. The number of particles $N_{\text {max }}$ included in each calculation and the relevant $\mathrm{rms}$ deviation $\sigma$ are also shown. See Section 6 for further discussion.

\begin{tabular}{rrrrrrrrrr}
\hline $10^{3} \tau$ & $10^{4} \epsilon$ & $a_{1}$ & $a_{2}$ & $a_{3}$ & $10 a_{4}$ & $10^{2} a_{5}$ & $10^{4} a_{6}$ & $N_{\text {Max }}$ & $\sigma$ \\
\hline 38 & 0 & -21.035 & 18.295 & -7.295 & 19.521 & -6.082 & 40.857 & 3009 & 8.904 \\
38 & 60 & 24.756 & -20.883 & 5.201 & 0.493 & 7.946 & -8.993 & 3009 & 5.758 \\
38 & 70 & 32.475 & -27.496 & 7.306 & -2.704 & 10.297 & -17.326 & 3009 & 5.297 \\
38 & 80 & 39.762 & -33.786 & 9.329 & -5.806 & 12.597 & -25.559 & 3009 & 4.834 \\
50 & 0 & -24.946 & 24.641 & -10.384 & 26.117 & -13.000 & 82.558 & 2008 & 7.328 \\
50 & 50 & 14.051 & -13.208 & 3.323 & 2.409 & 7.006 & 6.484 & 2008 & 5.286 \\
50 & 53 & 16.795 & -15.857 & 4.264 & 0.817 & 8.320 & 1.634 & 2008 & 5.175 \\
50 & 55 & 18.589 & -17.556 & 4.862 & -0.188 & 9.150 & -1.464 & 2008 & 5.098 \\
\hline
\end{tabular}

